\title{
Ciencia y tecnología en Colombia. Implicaciones para la educación
}

SCIENCE AND TECHNOLOGY IN COLOMBIA. IMPLICATIONS FOR THE EDUCATION

CIÊNCIA E TECNOLOGIA EM COLOMBIA. ENVOLVIMENTOS PARA A EDUCAÇÃO

Diego Hernán Arias Gómez* / dhariasg@udistrital.edu.co

\section{Resumen}

El presente escrito expone los recientes debates en torno a la regulación de los derechos de autor en la internet, en el contexto del llamado capitalismo cognitivo y los compromisos del país con el gobierno de los Estados Unidos. Realiza un repaso de conceptos como sociedad de la información, compromisos con el TLC y política pública en materia de las TIC. Finalmente identifica el papel asignado a la educación y la escuela en esta dinámica.

\section{Summary}

The written present exposes the recent debates concerning the regulation of the copyright in the Internet, in the context of the so called cognitive capitalism and the commitments of the country with the government of the United States. It realizes a revision of concepts as company of the information, commitments with the TLC and public politics as for the TIC. Finally he identifies the paper assigned to the education and the school in this dynamics.

\section{Resumo}

O presente escrito expõe os recentes debates em torno da regulação dos direitos de autor na internet, no contexto do chamado capitalismo cognitivo e os compromissos do país com o governo dos Estados Unidos. Realiza um repaso de conceitos como sociedade da informação, compromissos com o TLC e política pública em matéria das TIC. Finalmente identifica o papel atribuído à educação e a escola nesta dinâmica.

\section{Palabras clave}

Derechos de autor, capitalismo cognitivo, educación y tecnología

Key words

Copyright, cognitive capitalism, education and technology

Palavras chave

Direitos de autor, capitalismo cognitivo, educação e tecnologia

\footnotetext{
* Docente de la Universidad Distrital Francisco José de Caldas; estudiante del Doctorado Interinstitucional en Educación de la Universidad Pedagógica Nacional.
}

Fecha de recepción: 5 de mayo de 2012 / Fecha de aprobación 8 de junio de 2012 
Atravesamos una revolución tecnológica cuya peculiaridad no reside tanto en introducir en nuestras sociedades una cantidad inusitada de nuevas máquinas, sino en configurar un nuevo modo de relación entre los procesos simbólicos -que constituyen lo cultural- y las formas de producción y distribución de los bienes y servicios: un nuevo modo de producir, asociado a un nuevo modo de comunicar, convierte a la información y al conocimiento en fuerza productiva directa.

(Martín-Barbero, 2005, p. 24).

\section{Introducción}

En abril de 2010, el Ministro de Interior y Justicia de Colombia, Germán Vargas Lleras, presentó al Congreso una Ley antipiratería que pretendía preservar los derechos de autor en el entorno de la Internet. Si bien esta propuesta no prosperó para esa época, ella planteaba que un autor que creyera vulnerados sus derechos por una publicación en la red podía exigir remuneración ante la empresa prestadora del servicio de internet, entablar demandas judiciales y pedir, desde compensaciones económicas, hasta la baja de su material en la Web.

Entre las numerosas y polémicas posiciones a favor y en contra llamó la atención la expuesta por Gustavo Palacio, Director de la Asociación para la Protección de Derechos Intelectuales sobre Fonogramas y Videogramas musicales -APDIF-, quien frente al señalamiento del proyecto como "inmaduro y apresurado" afirmó que era un:

\section{[...] craso error decir que esta ley no se ha dis-} cutido. Recoge las obligaciones que ya tenemos en el TLC con Estados Unidos y eso fue un tema de amplio debate. Se abrió la posibilidad para que los sectores privados intervinieran y así pasó (Palacio, 2011).

Meses después, en abril del 2012, de espaldas a la opinión pública, el gobierno colombiano impuso sus mayorías en el Congreso y se aprobó en tiempo record la Ilamada "Ley Lleras 2.0" como preámbulo para el arranque del TLC entre Colombia y Estados Unidos. Este Proyecto de Ley revive las pretensiones del 2010, en cuanto a la regulación de los derechos de autor y la propiedad intelectual en la Web. Por otro lado, de fecha más reciente, en los Estados Unidos, las leyes antipiratería conocidas como SOPA (Stop Online Piracy Act) y PIPA (Protect Intellectual Property Act) postuladas por las cámaras del Congreso, fueron detenidas parcialmente hasta nuevo aviso. Ello no impidió el cierre de la página Megaupload y la judicialización de su director por parte del FBI.

Más allá del folclor político que este tipo de medidas suscitan y de las medidas de protesta de Asociaciones, Google, Wikipedia y otros portales, dos cosas se ponen de relieve en este debate: la importancia de lo virtual, la Web y su masificación, y el valor de la demarcación, desde la política pública, de límites entre lo legal y lo ilegal, lo prohibido y lo permitido en esta esfera, en cuyo caso los derechos de autor apenas son una expresión visible del fenómeno. Además, la respuesta del gobierno colombiano pone en evidencia su voluntad para acatar los compromisos en el contexto internacional por encima de las necesidades del país.

Curiosamente, y en forma paralela, se adelanta en el Congreso colombiano otro Proyecto de Ley que busca establecer el acceso a internet como un derecho fundamental, con las correspondientes políticas públicas que permitan y garanticen su disponibilidad. Esta dinámica se da en el marco de la declaración de las Naciones Unidas, de junio de 2011, que postuló la internet como un derecho humano.

La esfera de los derechos, al igual que la de las políticas públicas, es elástica, se ensancha o se encoge de acuerdo a las pujas y a los intereses en juego. Las Ilamadas tecnologías de la información y la comunicación cobran en las últimas décadas creciente interés en el mundo político y social, y se han constituido en un campo estratégico de poder y control. Ellas aterrizan en el país y colonizan la cultura, el deporte, la economía, la educación y la política, entre otros. Llegaron para quedarse. El presente escrito busca dar cuenta de su presencia y direccionamiento en algunos documentos de la política pública nacional, bajo el entendido que ésta, al prescribir unas orientaciones sobre el papel de la ciencia y tecnología en la sociedad colombiana, contribuye a forjar un tipo de subjetividades proclive a ciertos intereses y necesidades. 


\section{Era de la información, el conocimiento y el control}

Lo tecnológico hace parte lo humano desde la historia misma del hombre en tanto pone en evidencia su condición de reinvención y creación permanente. Para Rueda (2004) "la máquina deja de ser una cosa que debe ser trabajada, animada y dominada, pues la máquina somos nosotros y los procesos tecnológicos un aspecto de nuestra encarnación" (p. 72). Sin embargo desde el siglo XX esta interrelación se ha radicalizado, complejizado y masificado de forma inusitada respecto a otros tiempos. Gracias a su creciente presencia, parece ser, las sociedades y buena parte de los sujetos que las habitan, producen, piensan, se relacionan y aman de una nueva forma.

El capitalismo cognitivo es el nombre que recibe el desarrollo de una economía basada en la difusión del saber y "en la que la producción de conocimiento pasa a ser la principal apuesta en la valorización del capital" (Vercellone, 2004, p. 66). Se corresponde con la actual fase, en la que la producción inmaterial e intelectual cobran relevancia y en la que las actividades que condensan alta intensidad de saberes (servicios informáticos, multimedia, software) se consideran como el componente clave que define el crecimiento y la competitividad de las naciones. Esta mutación evidentemente trastoca el modelo fordista de organización del trabajo y resalta aspectos que la economía tradicional había excluido como campos de investigación: la cultura, la comunicación, el lenguaje, la producción social del saber. Elementos sobre los que recaerá la producción de riqueza y los deseos de control político y económico.

Esta mutación en las lógicas de acumulación transforma, entre otras cosas, la división internacional del trabajo y el lugar de las relaciones entre el centro y la periferia de las naciones, entre los países pobres y los ricos, pues se resitúan y descentralizan las actividades productivas y se modifican las antiguas formas de dependencia y explotación. El trabajo intelectual no nació con el computador, pero mientras este tipo de trabajo en el pasado desempeñaba funciones externas al proceso productivo y obtenía de la renta los medios para su manutención, hoy produce valor gracias a la interacción con más trabajo de su misma índole y no gracias a la orientación y explotación de la fuerza de trabajo física (Berardi, 2003).
En este sentido, Vercellone (2004), afirma que "el desarrollo desigual de la economía del conocimiento tiende así a conducir a una lógica autosostenida y acumulativa que condena a un cierto número de países en desarrollo a una verdadera 'desconexión forzada'" (pp. 68-69). Dicha desconexión agudiza las antiguas formas de marginación socioeconómica al confinar a unos países a la especialización en actividades intensivas de mano de obra con débil contenido de conocimiento. La masificación de la formación técnica y tecnológica, entendida como la capacidad operativa del sujeto en detrimento del saber y la teoría que le subyace, constituye un buen ejemplo de este esfuerzo por reubicarse al margen en la división internacional del trabajo.

Otro eje en torno al cual se estructura la nueva división internacional del trabajo, tiene que ver con la propiedad del saber $y$, por ende, con los esfuerzos por establecer los derechos de propiedad intelectual que se esgrimen como condición esencial para la innovación, pues supuestamente este reconocimiento y cobro en dinero es el que le permite a las firmas paliar los rubros invertidos en tecnología. En realidad este "cercamiento del saber" es una de las condiciones de la nueva acumulación capitalista.

En este mismo sentido Lander (2001) considera que la defensa de este tipo de propiedad expresa la oposición entre los intereses de las grandes corporaciones transnacionales y las mayorías del sur. El autor muestra esta importante asimetría al denunciar cómo los tratados internacionales esgrimen unos regímenes de protección legales que no sólo legitiman el saber occidental, sino que la aplicación de éstos acentúa el monopolio y:

[...] protege [n] aquello en lo cual los países y transnacionales del Norte tienen ventajas, mientras que, básicamente, deja sin protección aquello en lo cual los países y pueblos del Sur tienen una indudable ventaja: en la diversidad genética de sus territorios y en el conocimiento tradicional de los pueblos campesinos y aborígenes (Lander, 2001, p. 85).

Para Rullani (2004), la articulación entre economía y conocimiento es de vieja data, quizá desde la revolución industrial, con la ciencia y la tecnología incorporadas a las máquinas y la organización científica del trabajo es que la sociedad hace extensivas sus capacidades de prever, programar y calcular los aspectos económicos y sociales a través del uso del conocimiento, en este 
sentido, el motor del capitalismo siempre ha sido este enfoque pragmático de conocimiento: "En el curso de los dos últimos siglos, el conocimiento ha jugado su papel en la objetivación del mundo, adaptando la naturaleza y los hombres a la producción" (p. 100).

Sin embargo, a diferencia del pasado, hoy el conocimiento sufre un proceso de virtualización al separarse de su soporte material, ello provoca muchas incomprensiones y perplejidades al no contar con referentes teóricos claros, pues son caducas las clásicas categorías de análisis. Valor de uso, valor de costo, valor de cambio, aplicados al conocimiento, generan definiciones y límites muy gaseosos difícilmente extraíbles de las antiguas concepciones ligadas a los bienes escasos y finitos propios de las reflexiones de la economía clásica liberal o marxista. Como ejemplo, hoy el valor de cambio, si bien materialmente tiende a cero, se disputa en la (im) posibilidad de limitar su libre difusión por medios jurídicos o institucionales gracias a patentes, licencias o contratos.

Sin bien las antiguas formas de producción configuraban formas específicas de dominación, y por tanto perfilaban subjetividades disciplinadas y afines a las lógicas de repetición, producción y acumulación, el actual capitalismo cognitivo también demanda formas de control y subjetividades funcionales a sus principios.

Lazzarato (2006), guardando distancia con las lecturas marxistas del mundo y la sociedad, y sirviéndose de Foucault y Deleuze, piensa que la dominación, el sometimiento de los cuerpos y la imposición de conductas no deben explicarse solamente como parte de las demandas económicas, pues:

[...] los regímenes de signos, las máquinas de expresión, los agenciamientos colectivos de enunciación (el derecho, los saberes, los lenguajes, la opinión pública, etc.) actúan como ruedas del resto de agenciamientos, del mismo modo que los agenciamientos maquínicos (fábricas, prisiones, escuelas) [En este sentido cobran relevancia las instituciones que se muestran como agentes de integración, de estratificación del poder y que a su vez se derivan de él, ya que] [...] Las relaciones de poder son virtuales, inestables, no localizables, o estratificadas, potenciales, y definen solamente las posibilidades, las probabilidades de interacción; son las relaciones diferenciales las que determinan las singularidades (Lazzarato, 2006, pp. 75-77).
Las instituciones efectúan el control mediante la diferenciación y la integración que, para Lazzarato, consisten en ligar singularidades, homogenizarlas y disponerlas hacia un objetivo común. La diferenciación consiste en el esfuerzo de clasificar y oponer las diversas fuerzas que emergen en lo social; es la creación y reproducción de dualismos que simplifican las dinámicas e imponen lecturas opuestas, disyuntivas de la realidad. Esto funciona como un dispositivo de captura y codificación de las múltiples combinaciones y fuerzas que suceden en la sociedad.

Para Deleuze se busca encerrar el afuera, lo virtual, el devenir, bajo la consideración de que las sociedades disciplinarias ejercen su poder neutralizando la diferencia y su potencia de variación subordinándola a la reproducción. "El adiestramiento de los cuerpos tiene por función impedir toda bifurcación, retirarle al acto, a la conducta, al comportamiento, toda posibilidad de variación, toda imprevisibilidad" (Lazzarato, 2006, p. 80). Las instituciones disciplinarias no se limitan a reprimir, producen subjetividad.

En los tiempos actuales, dada la imposibilidad de neutralizar el devenir y la diferencia, afirma Lazzarato, se impone el control, y a su vez las sociedades de control producen sus tecnologías y sus procesos de subjetivación. Si las sociedades disciplinarias modulan cuerpos, las de control además de éstos modulan los cerebros. Aquí las relaciones de poder se evidencian en las acciones a distancia de un espíritu sobre otro, por la capacidad mediatizada y enriquecida por la tecnología de afectar pensamientos y ser afectados por ellos. El control del público y la opinión pública se plantean como el caso por excelencia del control que opera en las nuevas sociedades, que no borra las antiguas adscripciones y pertenencias a la clase, el sexo, o la tradición, sino que las inscriben en una nueva matriz en las que unas y otras se ponen y sobreponen en complejas y flexibles relaciones de sentido.

En las sociedades de control el poder ocurre indirectamente y las técnicas de control actúan más sobre las reglas del juego que sobre el juego mismo. Dado que la acción del sujeto halla su fuente en el medio, es éste la fuente de intervención. El medio "es lo que se necesita para dar cuenta de la acción a distancia de un cuerpo sobre otro. Es entonces el soporte y el elemento de la circulación de una acción" (Foucault, citado por Lazzarato, 2006, p. 231). 
Recapitulando, es posible decir que, por diferentes circunstancias, nos hallamos frente a un nuevo tipo de entorno social y económico atravesado por lógicas tecnológicas e informacionales que ubican el lugar de la producción y la riqueza en un nuevo escenario que algunos autores han denominado capitalismo cognitivo, en el sentido en que el saber y el conocimiento se configuran como mediaciones para producir riqueza; además este tipo de capitalismo, en tanto sociedad de control, configura un nuevo tipo de subjetividad, unas relaciones de sentido y unas formas de regulación y neutralización de la diferencia y la repetición. Ahora bien ¿de qué manera la actual sociedad pretende el control sobre las nuevas tecnologías en Colombia?

\section{Colombia frente a las TIC}

El Plan Nacional de Desarrollo 2010-2014 (DNP, 2011) establece los compromisos y las perspectivas del Gobierno Nacional en las diferentes ramas de la sociedad, en particular, define sus metas de crecimiento y productividad para los próximos 4 años. El documento se refiere a 5 locomotoras que supuestamente impulsarán el crecimiento económico, reducirán la pobreza, la indigencia y el desempleo, una de ellas, la de la innovación, se vincula directamente a la generación de nuevas tecnologías: "Facilitar y fomentar el uso y adaptación de tecnología son requisitos fundamentales para que la innovación en el país evolucione hacia la frontera del conocimiento" (p. 55).

El Plan propugna por un uso intensivo de la tecnología y por su intervención en sectores estratégicos de la sociedad, le impone un gran valor, pues la vincula con el crecimiento económico y reconoce la deuda histórica del país en cuanto a la inversión en investigación y desarrollo tecnológico, también postula explícitamente la intención de articular la generación de conocimiento al capital humano. Esta valoración de la tecnología no se queda sólo en intenciones, sino que sugiere aspectos metodológicos para su aplicación, en concreto, el Gobierno busca trabajar en tres campos para saldar esta brecha: la financiación, la formación y la organización.

La financiación está dirigida a proyectos de investigación que beneficiarán a los sectores científico, tecnológico y productivo; habrá alianzas con el sector productivo e incentivos tributarios al sector privado. En este contexto, formar significa propender por la articu- lación del sector académico y productivo y garantizar la apropiación social de la ciencia y la tecnología, además se postula el otorgamiento de becas posgraduales. Acá como en el anterior punto hay un énfasis en la relación con la empresa, la formación técnica y tecnológica, pasantías e intercambios "con capacidad para transformar ideas y conocimiento en nuevos o significativamente mejorados productos, servicios o nuevos procesos en el mercado" (DNP, 2011, p. 62). El modelo para implementar prototipos que articulen procesos de educación y emprendimiento serán las tecno-academias del SENA.

Por último, la organización tiene que ver con la reestructuración del Sistema Nacional de Ciencia, Tecnología e Innovación -SNCTel-, con la reorientación del Colciencias, que se apoyará en el esquema tripartito de Estado-Sector privado-Academia y con los esfuerzos por ampliar lo que viene haciendo el SENA. Llama la atención, en el documento, la combinación de un fuerte interés por la apropiación de la tecnología en diferentes esferas de la sociedad, por su organización y financiación, junto a la reiteración del mercado como instancia con la cual se relaciona, articula, vincula o supedita la tecnología en el país.

Sobre el uso de las Tecnologías de las Información y las Comunicaciones -TIC-, el Plan Nacional de Desarrollo establece que se estimulará su uso sobre todo en el sector productivo y se trabajará en la masificación de la banda ancha a través de las redes móviles. Se fortalecerá la investigación y la formación del capital humano en este campo. El carácter instrumental y el interés en función de la empresa de las TIC quedan ratificados al afirmar que:

Se consolidará a las TIC como plataforma tecnológica de los procesos educativos mejorando con ello la cobertura, la calidad, la pertinencia y la generación de contenidos educativos, con lo cual se fortalecerá la fuerza laboral en el uso de las TIC.

Se mejorarán las capacidades de los profesores de educación básica y media en TIC y se promoverá la incorporación del uso de estas tecnologías como herramientas académicas y educativas (DNP, 2011, p. 65). 
En la escuela, las TIC se conciben aquí como "plataforma", como "herramienta", es decir, piso o base sobre el que circulan otros dispositivos en función de alcanzar metas políticas, también como mero instrumento pedagógico que se inscribe en las lógicas convencionales. Esta apropiación de la tecnología en un escenario social tan supremamente estratégico como lo es la educación, demuestra la ambigüedad de la política pública que quiere ponerse a tono con el capitalismo cognitivo, ya que las generalidades sobre las bondades de las TIC que enuncia el Plan, se concretan en este campo en una aplicación reduccionista y simplista que a lo sumo parece que busca reemplazar el papel o la pizarra en tanto utensilios escolares.

Por otro lado, las peticiones de las agencias internacionales, también en el contexto del capitalismo cognitivo, en el sentido de preservar y salvaguardar los derechos de autor, se trasladan al Plan Nacional de Desarrollo, que define taxativamente compromisos en torno a la propiedad intelectual, a la que considera guardiana de la transmisión de la identidad cultural, la cohesión social y la calidad de vida.

El Plan estipula que los derechos de propiedad intelectual crean incentivos para la inversión extranjera y para la confianza de los consumidores, lo que en últimas -según el texto- redunda en crecimiento económico. Para ello se fortalecerá la capacidad de la propiedad industrial con aumento de presupuesto, se pulirán los mecanismos de seguimiento y control, se fortalecerá la Dirección Nacional de Derechos de Autor (DNDA) y se fortalecerán los espacios de coordinación de políticas de las distintas entidades a través de la Comisión Intersectorial de Propiedad Intelectual (CIPI); además, se implementarán programas de educación sobre el respeto a los derechos de autor y campañas en el sector público y privado que propendan por este objetivo.

En este punto el Plan se compromete, dentro de sus metas, a pasar de 30 a 100 grupos de investigación con patentes reconocidos por Colciencias, modelos de utilidad u obtenciones vegetales otorgadas. Estas políticas estatales se ajustan al funcionamiento de una economía basada en la producción de conocimiento, en la que éste genera un valor económico en el contexto de los bienes privados señalando la distancia entre usuarios y productores. La consecuencia es que el conocimiento se convierte en algo exclusivo de quienes pueden acceder económicamente a él y ya no de quienes lo producen. Así, universidades y centros de investigación, por ejemplo, se convierten en empresas que compiten en un mercado.

Por su parte la Ley de Ciencia y Tecnología, ley 1286 de 2009 (Congreso, 2009), reforma el Sistema Nacional de Ciencia y Tecnología, transformando a Colciencias en el Departamento Administrativo de Ciencia, Tecnología e Innovación. Se busca la incorporación de la ciencia y la tecnología como ejes transversales de la política económica y social del país. Este ente será el que coordine el Sistema Nacional de Ciencia, Tecnología e Innovación (SNCTI) y quien defina los gastos del Fondo Nacional de Financiamiento para la Ciencia, la Tecnología y la Innovación, Fondo que a su vez canalizará los recursos públicos y privados, nacionales e internacionales y las donaciones orientadas a la financiación de actividades de ciencia y tecnología.

La autonomía de este nuevo departamento queda en cuestión, pues, según la Ley, tendrá un Consejo Asesor que estará completamente integrado por personas designadas por el Presidente de la República (el Director, 4 Ministros, el Director del DNP, el Director del SENA, 4 personas del sector académico, 4 del sector productivo y 2 del sector científico regional). No se da la presencia de un solo sujeto independiente o de un sector o grupo que no sea nombrado por el Ejecutivo.

Además, la Ley define los siguientes criterios para el fomento y el estímulo de las actividades relacionadas con la ciencia, la tecnología y la innovación: evaluación para ponderar apoyos posteriores; participación de diferentes sectores en la toma de decisiones; descentralización territorial; revisión y actualización para garantizar el impacto en el aparato productivo; transparencia en el sentido de selección mediante convocatorias; continuidad, oportunidad y suficiencia; divulgación sin perjuicio de los derechos de propiedad intelectual; y protección, que también tiene que ver con los mecanismos para el reconocimiento de la propiedad intelectual.

El asunto con Colciencias y su articulación a sistemas globales de ciencia, tecnología e innovación es muy complejo, en gran medida porque al final todo este proceso está llevando a la conversión de las universidades en instituciones pragmáticas de cara a procesos de 


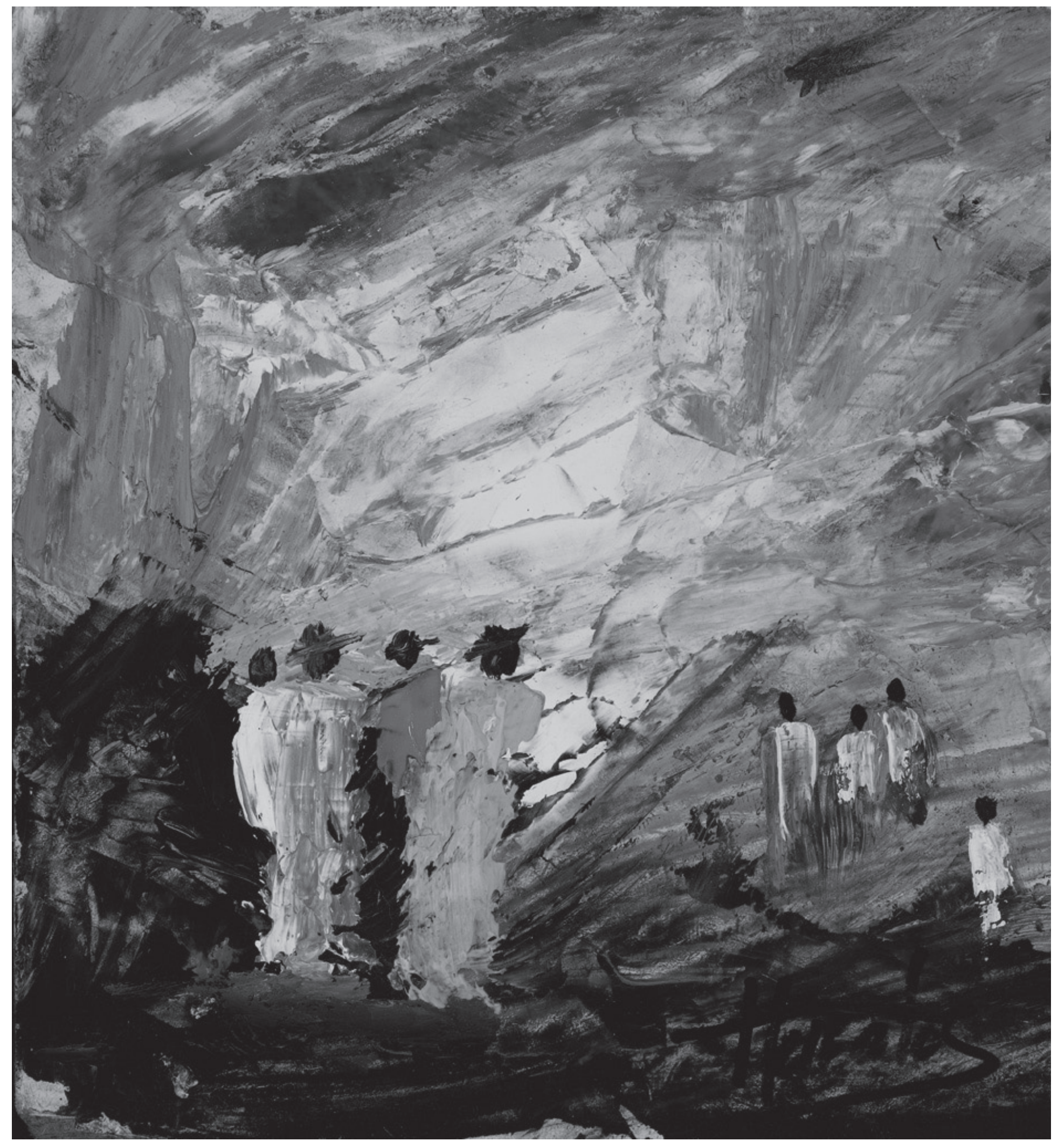

Segundo Arístides Huertas Torres ॥ Título: Lejania ॥Técnica: Óleo sobre tela ॥ Dimensiones: $80 \mathrm{~cm}$ x 60 cm 
evaluación externos e internos (de programas académicos, de artículos para revistas, de proyectos, etc.) y de procesos de conformación de comunidades globales de investigación que, en aras de una gestión internacional del conocimiento y de su métrica, pasan por encima de procesos y condiciones locales de producción.

Así mismo, esta tendencia a la mercantilización de la ciencia, por la alianza con la empresa privada, hace que enfrentemos cada vez más casos de "temas de moda", debido a un financiamiento externo que los posiciona en el ámbito nacional e internacional y los convierte en un producto de conocimiento para rentabilizarlo en el mercado, antes que considerarlos objetos de investigación y producción fruto de criterios de contenido científico o de pertinencia social y local.

\section{Educación, comunicación y tecnología}

El capitalismo cognitivo criollo se hace realidad en el Plan Nacional de Desarrollo en mención, que entiende el mejoramiento de la calidad de la educación como el desarrollo de las Ilamadas competencias. Para el documento, el capital humano se fundamenta en el desarrollo de competencias laborales y básicas que incluyan por una parte, el uso y apropiación de los medios y tecnologías de la información y la comunicación, y por otra, el manejo de una lengua extranjera que:

[...] en conjunto, le permiten a los ciudadanos enfrentar los retos y la competitividad propias del siglo XXI [...] Para esto, los procesos de enseñanza-aprendizaje basados en competencias tienen el potencial de mejorar el vínculo entre educación y trabajo, estableciendo nuevas vías desde la educación hacia el empleo y reduciendo algunas barreras del aprendizaje (aprender haciendo), por ejemplo al utilizar nuevas formas de evaluación. Estos procesos permiten que las personas estén preparadas para obtener, adaptar y aplicar la información en múltiples contextos y redes y transformarla en un conocimiento relevante para la satisfacción de sus necesidades de manera más equitativa, respetuosa con el ambiente y las distintas realidades, constituyéndose así en miembros productivos de la comunidad y de una sociedad cada vez más integrada a la economía global, con mayor bienestar. Así, desarrollan y fomentan la creación de mecanismos de certificación adecuados para que la conexión entre el sistema de formación y el desarrollo de los perfiles ocupacionales sea flexible y bidireccional (DPN, 2011, p. 86).

En este marco, la pertinencia está centrada en la correspondencia entre el sector productivo y el sector educativo, garante del desarrollo económico. También el uso de las TIC está presentado para que las instituciones desarrollen contenidos educativos en múltiples formatos y plataformas, además se garantizará la infraestructura tecnológica para el trabajo entre pares y se continuará con la promoción de su uso en las prácticas pedagógicas.

La formación docente pasará por la capacitación en competencias digitales, el diseño de ambientes virtuales de aprendizaje y la formación virtual para las regiones, para lo cual será de mucha utilidad el apoyo de las facultades educación del país y, por puesto, del SENA. Las metas hablan del paso, para el 2014, de 20 estudiantes promedio por computador conectado, a 12; mientras, se aumentará del $87 \%$ al $90 \%$ el porcentaje de estudiantes con conexión a internet de banda ancha las 24 horas desde la sede educativa; y habrá un crecimiento 194 a 300 en los programas académicos de pregrado y posgrado a distancia con $80 \%$ de virtualidad.

Adicionalmente hay que ver que estos procesos formativos se producen en un escenario complejo de mercantilización de los productos de conocimiento y de sus propósitos sociales de formación, alimentando el actual capitalismo, a la vez que lo reproduce y perpetúa en el seno de las diversas instituciones educativas. La extensión de la forma-empresa a todas las manifestaciones de la vida humana.

En síntesis, tanto la Ley de Ciencia y Tecnología, como el Plan Nacional de Desarrollo 2010-2014, expresan claramente el posicionamiento de la tecnología en la política pública nacional al apuntalar las TIC y el conocimiento como condiciones del crecimiento económico, pese a que no los define en concreto y más bien los homologa a la presencia de aparatos e internet.

Esta intención de abrazar el conocimiento y la tecnología por sí solos como fuente de felicidad y progreso, ha sido suficientemente desvirtuada por Berardi (2003), quien ha revelado las razones de la crisis del capital financiero a principios del 2000 y la importancia de entender todo crecimiento apoyado en la tecnología 
sobre la base del desarrollo material y estructural de la sociedad, destacando que: "el desarrollo no crea automáticamente riqueza para todos. De momento lo que se observa es la tendencia a la separación de dos humanidades diferentes en experiencia, conocimiento y renta" (p. 135). Lo que se está demostrado es que el nuevo orden internacional del trabajo y la riqueza cuenta con un nuevo catalizador, la tecnología, que recrea las viejas lógicas de explotación.

Además, las leyes colombianas hacen tangibles las pretensiones de acatar las prerrogativas internacionales por los derechos de autor, dándose una apropiación privada de los saberes que se sobrepone sobre los intereses colectivos. Estas prácticas, según Lander (2001), son impulsadas desde la Organización Mundial del Comercio en favor de la preservación de las ventajas de las ganancias de los países con altos niveles de industrialización y desarrollo tecnológico, hecho que se demuestra con el ínfimo número de patentes que históricamente han estado en manos de países pobres.

Finalmente, se destaca el papel que cumplen la educación y la escuela dentro de estas lógicas oficiales, al funcionar como escenarios privilegiados para implementar las dinámicas ligadas al conocimiento, la tecnología y los derechos de propiedad. Con esto se

\section{Referencias}

Berardi, F. (2003). La fábrica de la infelicidad. Nuevas formas de trabajo y movimiento global. Madrid: Traficantes de sueños.

Congreso de Colombia. (2009). Ley 1286 de 2009. Bogotá: Imprenta Nacional.

DNP. (2011). Bases para el Plan Nacional de Desarrollo 20102014. Más empleo, menos pobreza y más seguridad. Bogotá: Departamento Nacional de Planeación.

Lander, E. (2001). Los derechos de propiedad intelectual en la geopolítica del saber de la sociedad global del conocimiento. Comentario Internacional. Revista del Centro Andino de Estudios Internacionales, 2, II semestre. Quito: Universidad Andina Simón Bolívar.

Lazzarato, M. (2006). Por una política menor. Acontecimiento y política en las sociedades de control. Madrid: Traficantes de sueños.

Martín-Barbero, J. (2005). Cultura y nuevas mediaciones tecnológicas. En AA.VV. América Latina, otras visiones desde la cultura. Bogotá: Convenio Andrés Bello. demuestra que la escuela pervive como institución que materializa las intenciones políticas y pedagógicas del Estado y, en últimas, de entes multilaterales. Sumado a esto, se debe resaltar cómo en estas iniciativas se sobrepone una mirada reduccionista e instrumental de la tecnología, pues no la asume como un nuevo lenguaje y una nueva cultura que produce nuevas relaciones, nuevas subjetividades y riquezas a partir de otros códigos en la sociedad contemporánea, sino que más bien la aborda como asunto de herramientas a diseminar y adaptar, y que de paso define su éxito por el vínculo que se pueda generar con la empresa.

Frente a este aciago panorama oficial sólo resta decir que "sometida a la lógica del beneficio, la técnica se convierte en multiplicador de miseria" (Berardi, 2003, p. 158). Ello no impide reconocer la existencia de movimientos, grupos, portales, blogs, ONGs y expresiones de diversa naturaleza, que están realizando variadas formas de resistencia, en las que además de propender por el libre acceso al conocimiento, hacen visible en la Web las narrativas de sus prácticas organizativas, sus denuncias, sus apuestas y sus formas de construir subjetividad. La disputa por el acceso, consumo y producción en el mundo tecnológico está al orden del día.
Palacio, G. (2011). Polémica Ley Lleras. El Espectador. Obtenido el 19 de Octubre de 2011, desde, http://www.elespectador.com/ impreso/vivir/articulo-261793-polemica-ley-lleras

Rueda, R. (2004, Octubre). Tecnocultura y sujeto cyborg: esbozos de una tecnopolítica educativa. Revista Nómadas, 21. Bogotá: Universidad Central.

Rullani, E. (2004). Capitalismo cognitivo ¿un déjà vu?. En AA.VV. Capitalismo cognitivo: propiedad intelectual y creación colectiva. Madrid: Traficantes de sueños.

Vercellone, C. (2004). Las políticas del desarrollo en tiempos de capitalismo cognitivo. En AA.VV. Capitalismo cognitivo: propiedad intelectual y creación colectiva. Madrid: Traficantes de sueños. 


\section{Diálogo del conocimiento}

El debate contemporáneo de la producción de saber desde las nuevas tecnologías de la información y la comunicación, su regulación a partir de las políticas oficiales del gobierno colombiano, hace parte del tema central del presente artículo en donde además, el autor relaciona dicha situación con las nuevas dinámicas de producción capitalista y su impacto en la educación particularmente en la institución escolar.

El documento se encuentra estructurado por tres apartados, el primero de ellos nos ofrece un análisis de las llamadas sociedades de control desde autores como Guilles Deleuze y Mauricio Lazzaratto y Michel Foucault, quienes desde una perspectiva crítica de las sociedades modernas, ofrecen un análisis de las sociedades posfordistas de control y en donde efectivamente se evidencian transformaciones en las formas de producción capitalista, centradas en la producción inmaterial en donde la comunicación juega un papel fundamental en la constitución de públicos, de opinión pública que configuran formas de subjetividad contemporáneas.

En la segunda parte del documento, el profesor Arias centra su análisis en el lugar de la producción de saber desde las llamadas Tecnologías de la Información y la Comunicación en el marco de la llamada política pública colombiana, donde el autor acude a la Ley de Ciencia y Tecnología y al actual Plan Nacional de Desarrollo en tanto fuentes primarias y sobre las que intenta demostrar la visión tecnocrática e instrumental de la política oficial colombiana frente a la producción de conocimiento, cada vez más sujeta a la lógica mercantil.

El último apartado del artículo nos ofrece una reflexión sobre el lugar de la educación y particularmente la escuela en éste complejo escenario en el que la apuesta por la formación de capital humano desde el enfoque de las competencias, hace visible lo que para el autor es el capital cognitivo en la escuela. Allí la pregunta por el maestro, el lugar de su práctica pedagógica se hace fundamental en un escenario educativo subordinado a las lógicas de producción capitalista que reconfigura geográficamente la división social del trabajo y desde ésta perspectiva los lugares de producción de conocimiento. En ese sentido se sugiere prestar atención apropósito de prácticas alternativas y de resistencia por las que apuestan los maestros en diferentes regiones del país, condiciones de posibilidad para la aparición de otros saberes desde la colectividad.

Finalmente, el artículo provoca los siguientes interrogantes: para nuestras sociedades latinoamericanas y particularmente para la colombiana ¿Qué entendemos por conocimiento en el llamado capitalismo cognitivo? ¿Cuál es el lugar de los saberes como el de las ciencias humanas, que al decir de Barbero no pertenecen al grupo de los hoy llamados saberes rentables? ¿No es el trabajo inmaterial un trabajo que reposa sobre la pauperización del trabajo material de la mayoría de nuestra población, incluso sobre la pauperización de las condiciones materiales de los maestros en nuestro país? ¿Cómo reconfigurar desde la universidad éstas nuevas formas de producción de subjetividad y la subordinación del saber a la lógica mercantilista?

Víctor Manuel Rodríguez Murcia 